\title{
Protoplasmic Astrocytes in CA1 Stratum Radiatum Occupy Separate Anatomical Domains
}

\author{
Eric A. Bushong, ${ }^{1,3}$ Maryann E. Martone, ${ }^{1,2}$ Ying Z. Jones, ${ }^{1,2}$ and Mark H. Ellisman ${ }^{1,2}$ \\ ${ }^{1}$ National Center for Microscopy and Imaging Research, ${ }^{2}$ Department of Neurosciences, and ${ }^{3}$ Biomedical Sciences \\ Graduate Program, University of California, San Diego, La Jolla, California 92093-0608
}

\begin{abstract}
Protoplasmic astrocytes are increasingly thought to interact extensively with neuronal elements in the brain and to influence their activity. Recent reports have also begun to suggest that physiologically, and perhaps functionally, diverse forms of these cells may be present in the CNS. Our current understanding of astrocyte form and distribution is based predominately on studies that used the astrocytic marker glial fibrillary acidic protein (GFAP) and on studies using metal-impregnation techniques. The prevalent opinion, based on studies using these methods, is that astrocytic processes overlap extensively and primarily share the underlying neuropil. However, both of these techniques have serious shortcomings for visualizing the interactions among these structurally complex cells. In the present study, intracellular injection combined with immunohistochemistry for GFAP show that GFAP delineates only $\sim 15 \%$ of the total volume of the astrocyte. As a result, GFAP-based images
\end{abstract}

Protoplasmic astrocytes are the major glial cell of CNS gray matter. They are thought to play many important and diverse roles, including guiding development (Hatten and Mason, 1990; Ullian et al., 2001), regulating the extracellular concentrations of ions, metabolites, and neurotransmitters (Walz, 1989; Vernadakis, 1996), and supporting neuronal and synaptic function (Keyser and Pellmar, 1994; Araque et al., 1999). Protoplasmic astrocytes appear to be among the most structurally intricate cells of the brain. Certainly, the structure of astrocytes is intimately related to their functioning.

It has not been clearly determined how the complex morphology of astrocytes in vivo impacts the arrangement of astrocyte arrays. Wolff and colleagues approached the issue of interastrocytic relationships using what is known of astrocyte morphology from metal-impregnation and HRP-staining techniques (Wolff, 1976; Rohlmann and Wolff, 1996). They calculated that cortical astrocytes must interdigitate extensively. This conclusion was based on the observation that the extent of the average astrocyte is approximately spherical and that the average intersomal distance between astrocytes is approximately equal to the radius of the average astrocyte. Consequently, protoplasmic astrocytes are

Received July 26, 2001; revised Oct. 11, 2001; accepted Oct. 11, 2001.

This work was supported by National Institutes of Health Grants RR04050 from National Center for Research Resources and DC03192 from the National Institute on Deafness and Other Communication Disorders. We thank Dr. Diana MartinezPrice for her helpful discussions and for proofreading the manuscript. We also thank John Crum, Tom Deerinck, Stephan Lamont, and Dr. Stephen Young for their technical assistance.

Correspondence should be addressed to M. H. Ellisman, National Center for Microscopy and Imaging Research, 9500 Gilman Drive, La Jolla, CA 92093-0608. E-mail:mark@ncmir.ucsd.edu.

Copyright (C) 2001 Society for Neuroscience $\quad 0270-6474 / 01 / 220183-10 \$ 15.00 / 0$ have led to incorrect conclusions regarding the interaction of processes of neighboring astrocytes. To investigate these interactions in detail, groups of adjacent protoplasmic astrocytes in the CA1 stratum radiatum were injected with fluorescent intracellular tracers of distinctive emissive wavelengths and analyzed using three-dimensional (3D) confocal analysis and electron microscopy. Our findings show that protoplasmic astrocytes establish primarily exclusive territories. The knowledge of how the complex morphology of protoplasmic astrocytes affects their 3D relationships with other astrocytes, oligodendroglia, neurons, and vasculature of the brain should have important implications for our understanding of nervous system function.

Key words: glia; contact spacing; hippocampus; dye injection; Lucifer yellow; GFAP envisioned as possessing a very limited "autocontrol space", (i.e., the neuropilar volume in which they are the sole astrocyte) (Rohlmann and Wolff, 1996).

The distribution of astrocytes has been assessed commonly by labeling for glial fibrillary acidic protein (GFAP) (Eng et al., 1971; Bignami et al., 1972), an intermediate filament expressed exclusively by protoplasmic and fibrous astrocytes in the CNS. Previous studies have used GFAP to investigate the spatial relationships occurring between astrocytes in three-dimensional (3D) arrays in situ (Distler et al., 1991). These studies concluded that astrocyte somata achieve a nonrandom, region-dependent degree of spacing between themselves throughout the brain and, furthermore, that the processes of each astrocyte are extensively intermingled with those of neighboring astrocytes. The contacts occurring between processes were proposed to impart structural integrity to the nervous tissue while simultaneously separating neighboring astrocyte somata during development, allowing the resultant 3D array of astrocytes to effectively fill the nervous tissue. This phenomenon was termed "contact spacing" and was seen to be analogous to the "contact inhibition" phenomenon seen in vitro between fibroblasts (Dreher et al., 1994).

Recent studies have begun to suggest that heterogeneous populations of protoplasmic astrocytes may exist within hippocampal CA1 (Walz, 2000). Inconsistencies in GFAP immunoreactivity and in electrophysiological properties have been reported among astrocytes within this discreet region (Jabs et al., 1997; D'Ambrosio et al., 1998; Walz and Lang, 1998). The implications of these findings may not be fully realized unless we have an accurate understanding of interastrocytic spatial relationships (i.e., how do astrocytes distribute the neuropil among them- 
selves?). However, the approaches described above have left much unanswered. GFAP has long been known to grossly underestimate the full extent of astrocytes (Maxwell and Kruger, 1965; Connor and Berkowitz, 1985). Furthermore, conclusions resting on "average" astrocyte morphology may be inaccurate. Here we use dye injections in fixed tissue to study the spatial relationships between fully represented astrocytes in CA1.

Parts of this work have been published previously in abstract form (Bushong et al., 1999).

\section{MATERIALS AND METHODS}

Intracellular fills of astrocytes with fluorescent dyes in fixed tissue. The method for filling cells in fixed tissue slices was adapted from previously reported protocols (Buhl, 1993; Belichenko and Dahlström, 1995). Male Sprague Dawley rats, 1 month of age, were anesthetized with an overdose of Nembutal $(10 \mathrm{mg} / 100 \mathrm{gm}$ body weight) and perfused transcardially with oxygenated Ringer's solution at $37^{\circ} \mathrm{C}(0.79 \% \mathrm{NaCl}, 0.038 \% \mathrm{KCl}$, $0.020 \% \quad \mathrm{MgCl}_{2} \cdot 6 \mathrm{H}_{2} \mathrm{O}, 0.018 \% \mathrm{Na}_{2} \mathrm{HPO}_{4}, 0.125 \% \quad \mathrm{NaHCO}_{3}, 0.030 \%$ $\mathrm{CaCl}_{2} \cdot 2 \mathrm{H}_{2} \mathrm{O}, 0.20 \%$ dextrose, and $0.020 \%$ xylocaine) for $\sim 30 \mathrm{sec}$, followed by $0.1 \mathrm{~m} \mathrm{PBS}, \mathrm{pH} 7.4$, containing $4 \%$ paraformaldehyde $\left(37^{\circ} \mathrm{C}\right)$. For electron microscopic studies, $0.1 \%$ glutaraldehyde was added to the fixative. The fixative was perfused through the body for $10 \mathrm{~min}$, at which point the brain was removed and cut on a vibratome into $100-\mu$ m-thick coronal slices. The slices were stored in ice-cold PBS and used within 48 hr. The slices were placed in cold PBS and viewed with an Olympus Optical (Melville, NY) BX50WI infrared differential interference contrast (DIC)/epifluorescent microscope, using a $60 \times$ water immersion objective. Sharp glass micropipettes were pulled on a vertical pipette puller (David Kopf Instruments, Tujunga, CA) using omega-dot capillary tubes (outer diameter of $1.00 \mathrm{~mm}$ and inner diameter of $0.58 \mathrm{~mm}$; resistances ranged between 100 and $400 \mathrm{M} \Omega$ ) and backfilled with $10 \mathrm{mM}$ Alexa Fluor 568 in $200 \mathrm{~mm} \mathrm{KCl,} 10 \mathrm{~mm}$ Alexa Fluor 488 in $200 \mathrm{~mm} \mathrm{KCl}$ (Molecular Probes, Eugene, OR), or 5\% aqueous dilithium Lucifer yellow CH (LY) (Calbiochem, La Jolla, CA). The astrocytes were identified by the distinctive size and shape of their soma. The somata were impaled, and the dye was injected into the cells by applying a $0.5 \mathrm{sec}$ negative current pulse $(1 \mathrm{~Hz})$ until the processes were completely filled. After several cells were filled in a tissue slice, the slice was placed in cold $4 \%$ paraformaldehyde-PBS for $\sim 30 \mathrm{~min}$. At this point, the slices could be coverslipped in Gelvatol (Harlow and Lane, 1988) or processed for immunohistochemical labeling.

Immunohistochemical labeling. Tissue slices were washed in $25 \mathrm{~mm}$ Tris-buffered saline, $0.8 \% \mathrm{NaCl}, \mathrm{pH} 7.4$ (TBS) for $30 \mathrm{~min}$. Slices were blocked in TBS containing $2 \% \mathrm{NaCl}, 3 \%$ normal donkey serum (NDS), $1 \%$ cold-water fish gelatin (CWFG), $1 \%$ bovine serum albumin (BSA), and $0.1 \%$ Triton $\mathrm{X}-100$ (TX) for $1 \mathrm{hr}$ at $4^{\circ} \mathrm{C}$. The slices were then incubated for $72 \mathrm{hr}\left(4^{\circ} \mathrm{C}\right)$ with guinea pig polyclonal anti-GFAP antibody (Advanced ImmunoChemical, Long Beach, CA) diluted 1:200 in working buffer (TBS containing $2 \% \mathrm{NaCl}, 0.3 \% \mathrm{NDS}, 0.1 \% \mathrm{CWFG}, 0.1 \%$ $\mathrm{BSA}$, and $0.25 \% \mathrm{TX})$. The slices were then washed three times for $10 \mathrm{~min}$ each in working buffer and then placed in working buffer $\left(4^{\circ} \mathrm{C}\right)$ containing 1:100 donkey anti-guinea pig IgG conjugated to Cy5 (Jackson ImmunoResearch, West Grove, PA). After $24 \mathrm{hr}$, the slices were washed three times for 10 min each in TBS and then coverslipped in Gelvatol.

Imaging and analysis of dye-filled astrocytes. The Gelvatol anti-fade mounting media was allowed to set overnight. The filled astrocytes were then visualized using confocal laser scanning microscopy. The imaging was performed either on a Bio-Rad (Hercules, CA) MRC1024 with a $63 \times$ oil immersion [numerical aperture (NA) of 1.4] Zeiss (Oberkochen, Germany) objective or on a Bio-Rad Radiance2000 microscope with a $60 \times$ oil immersion (NA of 1.4) Nikon (Tokyo, Japan) objective. Z-motor calibration was checked using $\mathrm{Z}$-series through $15 \mu \mathrm{m}$ fluorescent latex beads. Proper channel alignment was confirmed using z-series through both latex beads and Purkinje cell spines that had been filled with both Lucifer yellow and Alexa 568.

Image visualization and analysis was performed using the Bitplane software suite (Bitplane AG, Zurich, Switzerland). Manual segmentation of astrocyte domains was achieved using DepthAnalyze module by Bitplane. This program allows polygons to be drawn around regions of interest through a z-series and creates $3 \mathrm{D}$ objects from the resulting stack of polygons. In this way, the neuropilar volume (volume of neuropil infiltrated by a single astrocyte) of filled astrocytes was determined. For examining the extent of GFAP labeling in LY-filled astrocytes, back- ground signal was first removed from the datasets by thresholding, and then the GFAP (Cy5) and LY channels were searched for voxels containing signal from both labels using the Colocalization module in the Bitplane package. This procedure finds all voxels within a confocal volume containing signal intensities from both channels within a range of user-defined values for each channel, in which voxel dimensions are determined by the pixel size $(0.09-0.15 \mu \mathrm{m})$ and step size $(0.36-0.81$ $\mu \mathrm{m})$. The resulting voxels containing colocalization constituted the volume of GFAP in the dye-filled astrocyte. The volume of the astrocyte was then determined by counting voxels containing LY signal. In examining the boundaries between protoplasmic astrocytes, colocalization was measured between the Alexa 488 and Alexa 568 signals. Low levels of background signal were removed using a baseline-subtraction threshold. Regions of interdigitation between astrocytes were then visualized by running the colocalization routine before and after performing a Gaussian blur $(0.5 \mu \mathrm{m}$ filter width $)$ on the two channels.

All ranges reported for mean values are the SEM and SD are provided as indications of population variance.

Photooxidation of Lucifer yellow-filled protoplasmic astrocytes. To examine astrocytes at the electron microscopic level, dye-filled astrocytes were photooxidized as described previously (Deerinck et al., 1994). Briefly, $100-\mu \mathrm{m}$-thick fixed slices were prepared as described above, except that $0.1 \%$ glutaraldehyde was added to the fixative. Astrocytes were filled with LY and then placed in $2 \%$ glutaraldehyde-PBS for $30 \mathrm{~min}$ at $4^{\circ} \mathrm{C}$. The slices were briefly washed in PBS and then placed in PBS containing $0.38 \%$ glycine for $2 \mathrm{~min}$. They were again rinsed in PBS and then placed in oxygenated PBS containing $0.1 \%$ potassium cyanide and $0.15 \%$ diaminobenzidine (DAB). After incubating in the DAB solution for $5 \mathrm{~min}$, the LY-filled astrocyte was exposed to intense illumination using a $75 \mathrm{~W}$ xenon lamp and a fluorescein excitation filter. The DAB solution was periodically replaced with freshly oxygenated solution during the process of photoconversion. When LY fluorescence was extinguished and the astrocyte was distinctly brown, the illumination was terminated, and the slice was washed three times for $10 \mathrm{~min}$ in ice-cold PBS.

The slices were subsequently processed for electron microscopy using a microwave-based protocol (Giberson et al., 1997). Briefly, the slices were osmicated in PBS-buffered $1 \% \mathrm{OsO}_{4}$ using two $40 \mathrm{sec}$ exposures to microwave irradiation in a Pelco 3440 microwave oven (Ted Pella Inc., Redding, CA) containing two $375 \mathrm{ml}$ water loads. After a 2 min rinse in distilled water, the slices were dehydrated with an ethanolic series $(50$, 70,90 , and $100 \%$ ), followed by dry acetone (two times for $40 \mathrm{sec}$ of irradiation per solution). The slices were infiltrated with a solution of $50 \%$ acetone-50\% Durcupan ACM epoxy resin (Electron Microscopy Sciences, Ft. Washington, PA) during 15 min irradiation and then with $100 \%$ resin during three times for $10 \mathrm{~min}$ each of irradiation. The slices were placed between two mold-release-coated slides and left at $80^{\circ} \mathrm{C}$ for $48 \mathrm{hr}$. Semithin $(0.5 \mu \mathrm{m})$ sections were cut and placed on Formvarcovered slot grids. Grids were poststained with uranyl acetate and Sato lead, coated with 10 and $20 \mathrm{~nm}$ gold particles, and carbon coated. Tilt series through $\pm 60^{\circ}$ were acquired on film at $400 \mathrm{KeV}$ using a JEOL (Peabody, MA) 4000EX electron microscope, and tomographic volumes were reconstructed as described previously (Perkins et al., 1997). The program Analyze AVW (Biomedical Imaging Resource, Mayo Foundation, Rochester, $\mathrm{MN}$ ) was used to examine the tomographic volumes.

\section{RESULTS}

\section{Efficacy of GFAP immunolabeling in assessing the morphology of CA1 protoplasmic astrocytes}

To observe protoplasmic astrocytes in their entirety, astrocytes in lightly fixed slices of hippocampus were iontophoretically filled with LY. Because the tissue slices were relatively thin $(100 \mu \mathrm{m})$ and the morphology of the tissue was well preserved, it was possible to locate and inject a large sample of cells. The majority of cells found in CA1 stratum radiatum are glial in nature, with the relatively few interneurons being very distinctive in their morphology. Astrocytes were chosen for dye injection based on the shape (rounded to oval) and size (7-9 $\mu \mathrm{m}$ in diameter) of their soma. The vast majority of cells filled based on these visual criteria were protoplasmic astrocytes, as evidenced by their unique spongiform morphology and GFAP expression. Although protoplasmic astrocytes in CA1 are known to be extensively 

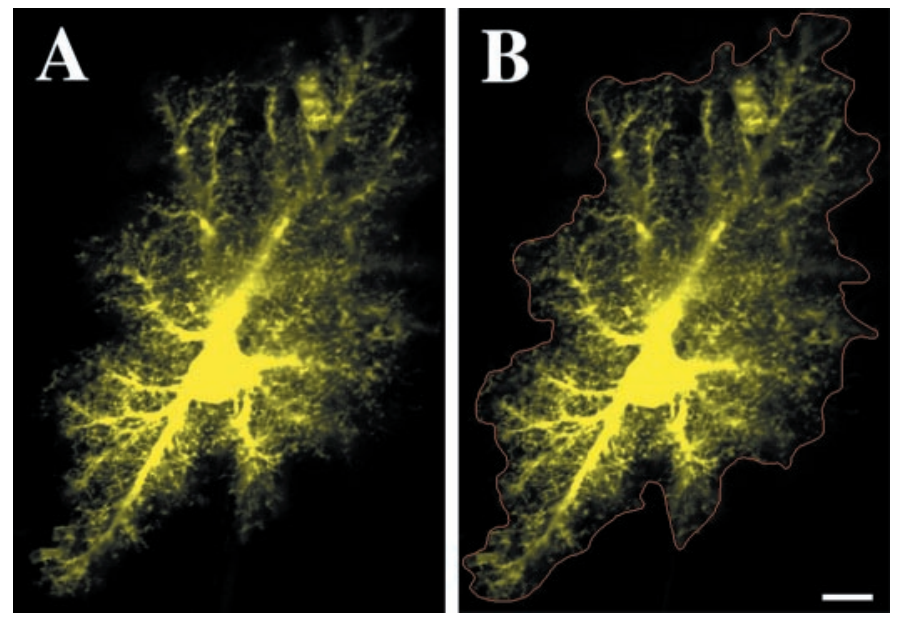

Figure 1. Protoplasmic astrocyte of CA1 stratum radiatum iontophoretically filled with the fluorescent dye Alexa 488. A, Optical slice reveals the dense spongiform processes of these astrocytes. A clear but complex boundary is evident in the extent of these processes. $B$, Via manual segmentation (red), it was possible to delineate the extent of and calculate the neuropilar volume occupied by each astrocyte. Scale bar, $10 \mu \mathrm{m}$.

coupled via gap junctions, aldehyde fixation blocks gap junctionalmediated dye transfer and enabled us to visualize individual cells.

Confocal microscopic volumes of the LY-filled astrocytes revealed the organization of the major processes and the distinctive spongiform ramifications belonging to each astrocyte (Fig. 1A). Wherever large branches extended outward from the soma, spongiform material consisting of very dense ramifications of fine processes was observed to extend $\sim 2-10 \mu \mathrm{m}$ out of these branches. The overall morphology varied greatly from astrocyte to astrocyte. The majority of astrocytes were approximately fusiform in shape, with their long axis oriented parallel to the descending apical dendrites of CA1 pyramidal cells, as described previously (Nixdorf-Bergweiler et al., 1994). However, it was not uncommon to find either spherical or markedly elongated astrocytes. The very dense nature of the processes of the astrocytes imparted a rather distinct boundary to the extent of each astrocyte. Only very rarely were fine thread-like processes seen to extend $5-10 \mu \mathrm{m}$ on their own beyond the boundaries defined by the rest of the spongiform material. These distinct boundaries allowed for the manual segmentation of the 3D extent of individual astrocytes through confocal volumes (Fig. 1B). Based on the resulting $3 \mathrm{D}$ segmented volumes, we calculated that the average astrocyte occupies a neuropilar volume of $65,900 \pm 3200 \mu \mathrm{m}^{3}$ $\left(n=20 ;\right.$ SD of $\left.14,500 \mu \mathrm{m}^{3}\right)$.

Immunolabeling of the hippocampal CA1 molecular layer for GFAP revealed numerous stellate structures distributed in a pattern similar to that observed in previous studies (SchmidtKastner and Szymas, 1990; Nixdorf-Bergweiler et al., 1994). When protoplasmic astrocytes were dye injected and then immunostained for GFAP, we observed characteristic labeling of individual astrocytes with GFAP (Fig. $2 A-C$ ). Astrocytes possessed 5-10 primary branches, from which extended several smaller side branches. The GFAP cytoskeleton generally radiated out from a central hub, which was sometimes composed of a triangular structure within the soma of the astrocyte (Fig. $2 A$ ). In optical sections, the radiating processes of neighboring astrocytes appeared to approach one another, without exhibiting considerable interdigitation, and GFAP filaments were very rarely seen to contact one another (Fig. $2 A$ ). It was considerably more difficult
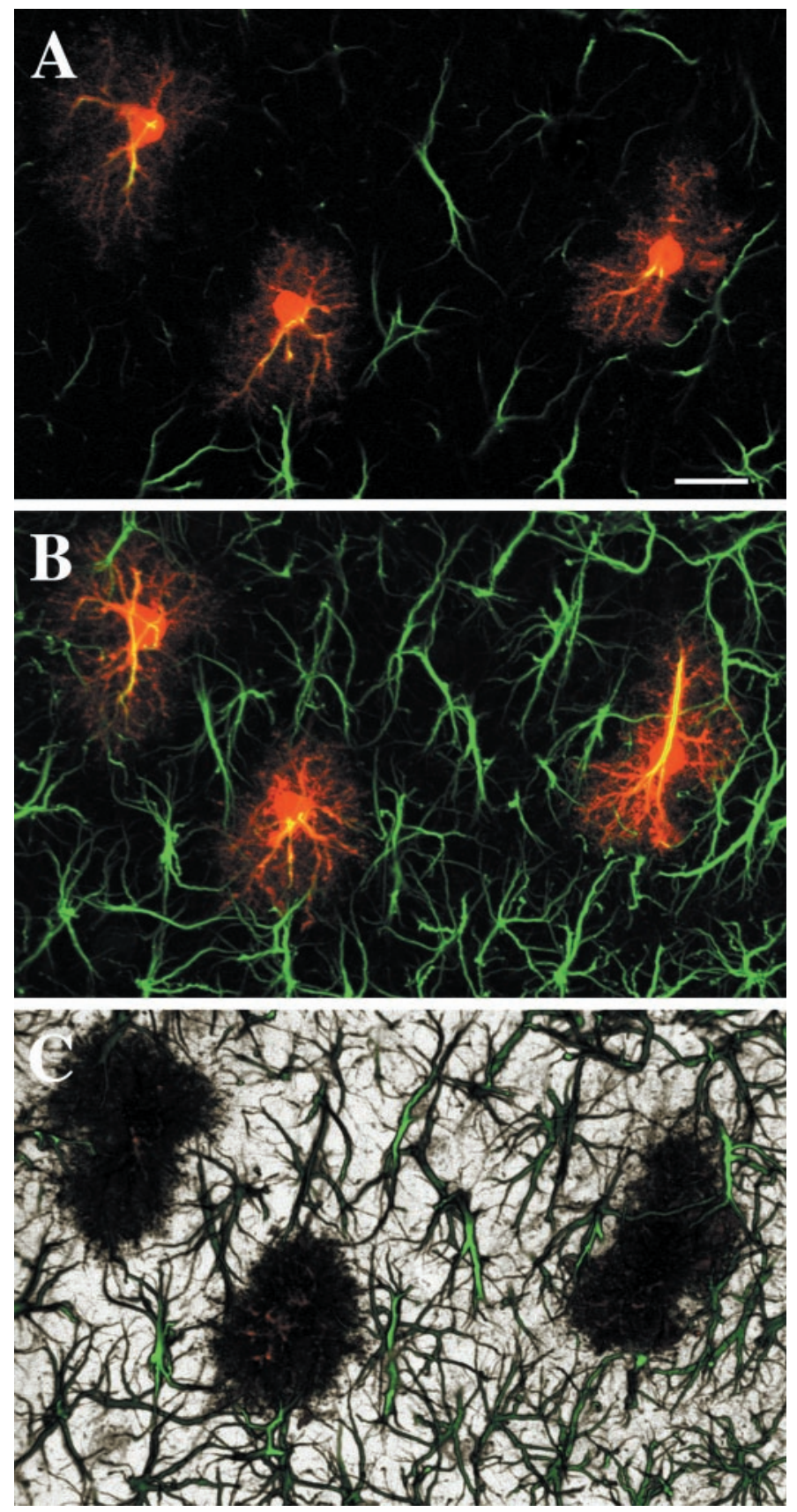

Figure 2. GFAP immunolabeling of LY-filled astrocytes in CA1 stratum radiatum. Dye-filled astrocytes (red) consistently display a GFAP cytoskeleton (green). A, An optical slice through double-labeled astrocytes reveals the limited degree of interdigitation or contacts between GFAP skeletons. $B$, A maximum projection through this volume of tissue $(\sim 25-$ $\mu$ m-thick) demonstrates the limited extent of GFAP throughout individual astrocytes. Relationships between GFAP skeletons are more difficult to resolve. $C$, In a $3 \mathrm{D}$ perspective projection through the same volume, the ability of astrocytes to fill space in the neuropil with their processes is apparent, as is the lack of lacunas in the felt work of GFAP processes, in which GFAP-negative variants of protoplasmic astrocytes could possibly reside. Volume thickness is $\sim 30 \mu \mathrm{m}$. Scale bar, $20 \mu \mathrm{m}$.

to determine the exact relationships between GFAP processes in maximum projections or $3 \mathrm{D}$ projections through volumes of labeled stratum radiatum, as seen in Figure 2, $B$ and $C$.

Some studies have suggested that subpopulations of protoplasmic astrocytes can be distinguished by a lack of GFAP 
staining. However, when 129 dye-filled cells, confirmed to be protoplasmic astrocytes based on morphology, were immunolabeled for GFAP, all 129 colabeled robustly for GFAP with the typical radiating pattern (Fig. $2 A, B$ ). Furthermore, 3D projections through the GFAP-labeled tissue showed that astrocyte somata and GFAP-positive processes were evenly distributed as described previously; no gaps were found in the labeling that might indicate that GFAP-negative protoplasmic astrocytes might be present (Fig. 2C).

To determine the extent of GFAP within an average astrocyte in CA1, protoplasmic astrocytes were filled with LY and subsequently immunolabeled for GFAP. The degree of extent of GFAP throughout individual astrocytes appeared to vary minimally. A colocalization module was used to estimate the extent of GFAP immunolabeling relative to total astrocyte volume in a random subset of these LY-filled astrocytes. It was determined that the volume of GFAP was $13 \pm 1 \%(n=9$; SD of $3 \%)$ of the total astrocytic volume, as revealed by LY. As expected, most of the GFAP was located in the soma, primary, and secondary processes, with very little GFAP found in the spongiform processes.

\section{Spatial relationships between neighboring protoplasmic astrocytes}

Because GFAP does not extend into the spongiform processes, it does not provide a good marker for examining the spatial relationships between neighboring astrocytes. To examine these relationships in detail, we filled 30 groups of astrocytes (each consisting of two to seven neighboring astrocytes) in the stratum radiatum of CA1 with a green fluorescent dye (either LY or Alexa 488) and a red fluorescent dye (Alexa 568). Under DIC optics, no astrocyte somata were seen to intervene between the filled cells. Optical sectioning through these groups of cells allowed us to discriminate between the processes of interacting astrocytes and examine their arrangement with respect to one another.

Examples of labeled groups of astrocytes are shown in Figures 3-7. Varying degrees of interdigitation were observed between the major processes of adjacent astrocytes. Direct interactions between neighboring astrocytes were mediated, however, through the intermingling of fine spongiform processes. Astrocytes appeared to be strongly influenced in their overall morphology by neighboring astrocytes (Fig. $3 A-C$ ). As seen in Figure 3, the interfacing boundaries of adjacent astrocytes often corresponded very well with each other, and the individual astrocytes appeared to occupy primarily distinct volumes of tissue. Often the interdigitation of the larger processes was quite limited, and the resulting segregation of the underlying neuropil between the neighboring astrocytes was especially dramatic. Indeed, in many instances, major processes appeared to avoid interdigitation or contact with a neighboring astrocyte. Larger processes were often seen to run tangential to the outer extent of an adjacent astrocyte, interacting with the adjacent astrocyte via their emanating spongiform processes (Fig. 4A). The intermingling between these processes at the interfaces between astrocytes appeared to occur within a very narrow region (Fig. $5 A$ ).

The somata of protoplasmic astrocytes generally appeared evenly separated from one another. However, the dendritic extensions of each astrocyte did not necessarily extend radially outward from each soma in all directions. Often the somata were highly displaced from the center of the overall extent of the astrocyte (Figs. $3 A, 4 B$ ). Large processes did not usually extend
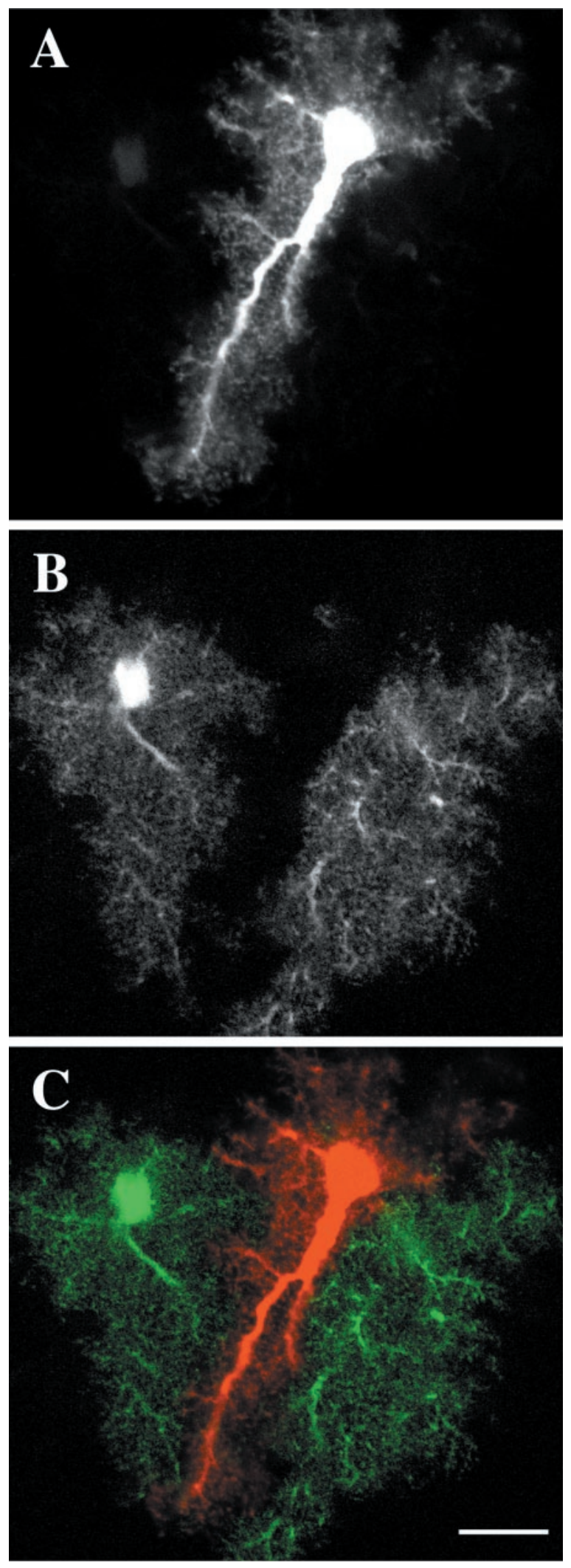

Figure 3. The overall morphology of astrocytes often appeared to be strongly influenced by their neighbors. $A$, Optical slice through an astrocyte filled with the Alexa 568. $B$, Alexa 488-filled astrocytes in the same optical slice. $C$, Overlay of $A$ and $B$. The astrocyte in the middle avoids overlap of its processes with its two neighbors. Scale bar, $15 \mu \mathrm{m}$. 

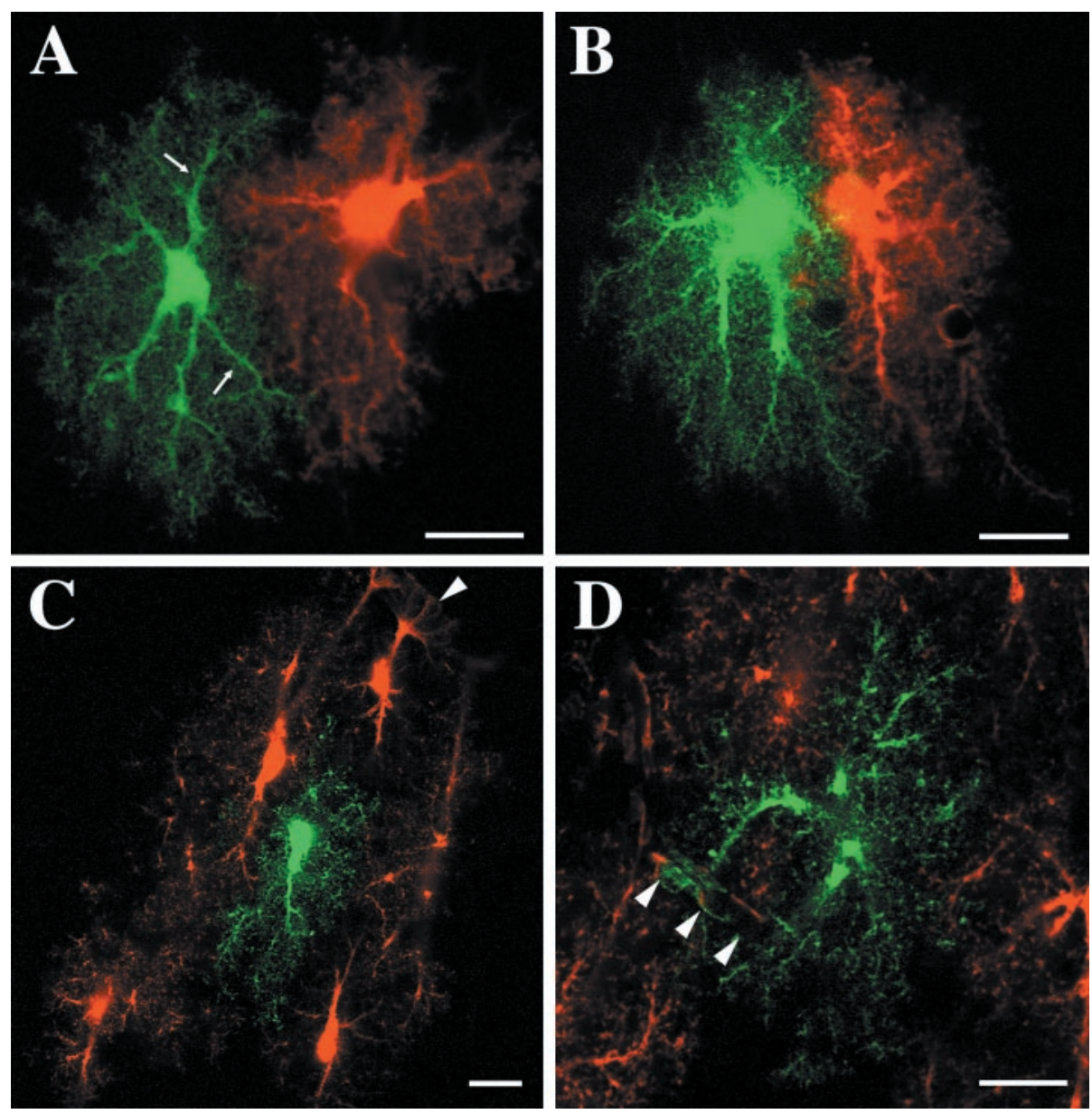

Figure 4. Optical slices through neighboring protoplasmic astrocytes filled with distinct fluorescent dyes. $A$, The major processes (arrows) of astrocytes were commonly seen to extend tangentially to the approaching processes of neighboring astrocytes. $B$, The processes emerging from two astrocytes with adjacent somata radiate parallel to or away from each other. $C$, Blood vessels (arrowhead) appeared to be capable of influencing the arrangement of astrocyte processes as they attempted to form end feet. Astrocyte on the far right is highly elongated as it reaches for passing vessel. The center astrocyte ( green) shows little overlap with its neighbors. $D$, One astrocyte (green) is seen to have its process "invade" the territory of its neighbor as both astrocytes form end feet on passing blood vessels. Scale bars, $15 \mu \mathrm{m}$.

completely to the soma of a neighboring astrocyte unless that soma did not itself extend processes in one direction, thereby allowing the process of its neighbor to approach. In such circumstances, the astrocytes usually extended their primary processes in opposite directions or parallel to each other (Fig. 4B). Spongiform process extending directly from the adjacent somata prevented direct contact between the somata.

Nearly every astrocyte formed end-feet processes with at least one blood vessel. Blood vessels appeared to influence the overall morphology of astrocytes, apparently prompting some astrocytes to develop decidedly longitudinal forms, presumably in an attempt to reach a vessel (Fig. 4C). Blood vessels also seemed capable of influencing interastrocytic interactions. The amount of interdigitation of astrocytic processes increased around blood vessels, as seen in Figure $4 D$, as if the astrocytes were competing for access to a passing blood vessel.

\section{Extensive overlap is observed between the processes of distinct glial cell types}

Occasionally, cells that exhibited the characteristic morphology of either oligodendrocytes or oligodendrocyte precursor cells in CA1 stratum radiatum were filled, possessing small, round somata and radial, varicose processes (D'Ambrosio et al., 1998; Levine et al., 2001). Cells of this type were less commonly found than protoplasmic astrocytes and did not stain for GFAP, and their somata were often in direct contact with the soma of a protoplasmic astrocyte or interneuron, a known characteristic of oligodendrocytes (Peters et al., 1991). When one of these oligoden- drocyte-like cells was filled next to a protoplasmic astrocyte, extensive interdigitation was observed among the processes of these distinct glial cell types (Fig. 5B). This suggests that, although the highly ramified nature of protoplasmic astrocytes appears to influence the morphology of other neighboring protoplasmic astrocytes and prevent them from encroaching into their space (Fig. 5A), it does not necessarily influence the extension of processes from other cell types. The conspicuous difference seen between the interactions of distinct cell types versus neighboring protoplasmic astrocytes suggests that the lack of interdigitation observed with the latter is not a result of our imaging or analysis techniques.

\section{Visualization of the interface region between astrocytes}

To enhance the visualization of the regions of contact between astrocyte processes throughout the $3 \mathrm{D}$ volumes, the volumes were searched for voxels containing both red and green signal using a colocalization routine. To highlight closely apposed processes at the interface zones, the astrocytes were first blurred slightly using a Gaussian blur filter. This process smeared the appearance of the fine processes and allowed for the detection of areas in which fine processes containing distinct dyes were interdigitated but of course not actually overlapping. Colocalization now effectively detected narrow bands of interaction occurring between astrocyte processes at the periphery of the extent of each astrocyte (Figs. 6, 7). When such volumes were viewed as $3 \mathrm{D}$ projections, they revealed a 

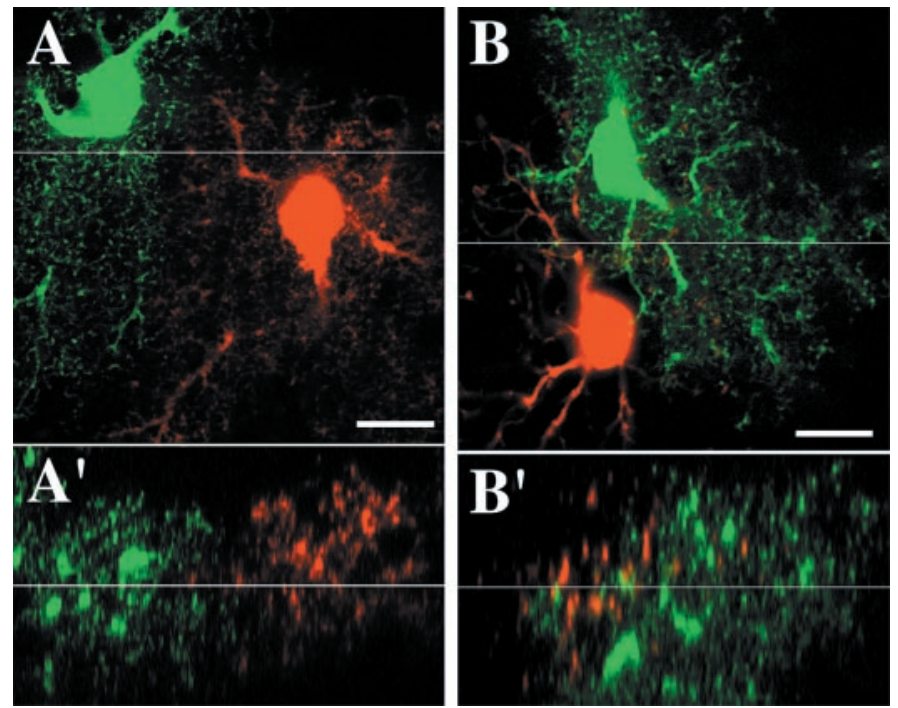

Figure 5. High-magnification view of interface region between the fine processes of neighboring astrocytes. $A$, Optical slice through the interface region between two adjacent protoplasmic astrocyte. Fine processes intermingle in a limited region at the interface zone. $A^{\prime}, x-z$ view of astrocytes in $A$. $B$, The processes of an oligodendrocyte-like cell (red) are seen to interdigitate extensively with the processes of an adjacent protoplasmic astrocyte. $B^{\prime}, x-z$ view of $B$. Scale bars, $10 \mu \mathrm{m}$.

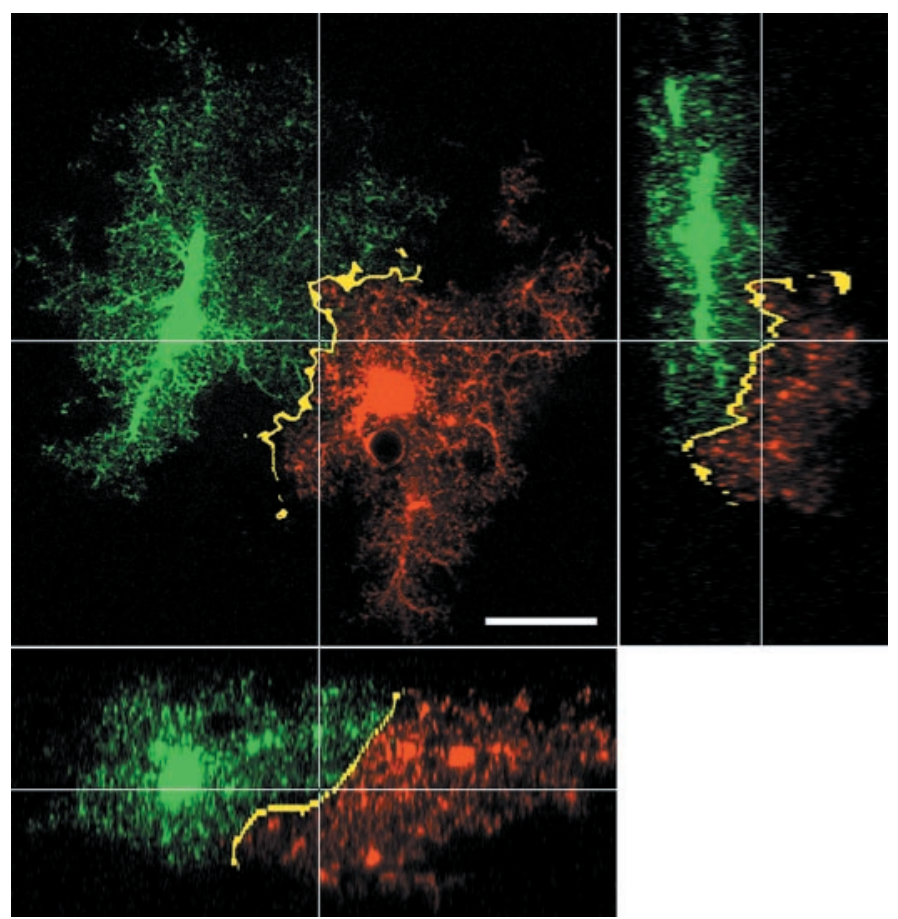

Figure 6. The discreet region of interaction (yellow) between the fine processes of protoplasmic astrocytes. Pixels containing both green and red were determined using the colocalization routine (see Materials and Methods) and then pseudocolored in bright yellow to mark their presence. $x-y$ (large panel), $x-z$ (bottom panel), and $y-z$ (right panel) slices through the area in which two adjacent astrocytes interface. Scale bar, $20 \mu \mathrm{m}$.

continuous, convoluted sheet-like zone of interaction between the neighboring astrocytes (Fig. $7 B$ ). The zone of interaction was extremely limited, with the bulk of the processes of the astrocytes occupying exclusive territories in the neuropil.

\section{Electron microscopic examination of the interface region between neighboring protoplasmic astrocytes}

Because the intermingling astrocyte processes at the borders of adjacent astrocyte domains are beyond the resolution of the light microscope, electron microscopy was used to examine the nature of the interactions in these regions. Protoplasmic astrocytes in CA1 molecular layer were filled with LY and photoconverted using fluorescence-based oxidation of DAB. When $0.5 \mu \mathrm{m} \mathrm{sec}$ tions were examined at low magnification $(1500 \times)$, the border regions of the spongiform material (in which the density of labeled astrocytic processes quickly diminished) were clearly visible, as was observed at the light microscopic level. At higher magnifications, electron tomography was used to create $3 \mathrm{D}$ reconstructions of the tissue at the apparent boundary of the extent of the labeled astrocyte. In the resulting volumes, even very fine processes (tens of nanometers in thickness) belonging to the filled astrocyte were discernable (Fig. $8 A, B$ ). The processes of the filled astrocyte appeared to extend to a certain point, beyond which it quickly became difficult to locate labeled processes. Presumably, the processes of the adjacent astrocyte are arranged very similarly, and the majority of interactions between the two cells occur only at the interface region. In Figure $8 A$, for example, a dendritic process can be seen to extend through the middle of the volume. On one side of the dendrite, there is a high density of labeled processes belonging to the filled astrocyte, whereas on the opposite side of the dendrite, no glial processes are labeled. Thus, the ultrastructural characteristics of the termination of a filled astrocyte are consistent with the conclusion derived from light microscopic examinations, i.e., a limited degree of overlap occurs between the extents of neighboring astrocytes, contrary to previous interpretations.

\section{DISCUSSION}

This is the first time that the spatial relationships between protoplasmic astrocytes have been studied when the astrocytes were visualized in their entirety. The observations made possible using this technique reveal an aspect of interastrocytic interactions until now underappreciated: each astrocyte solely contributes to the astrocytic component of neuropil in much larger regions than previously thought. The spongiform nature of these cells allows them to infiltrate distinct volumes of the neuropil, with interaction between neighboring astrocytes primarily confined to their borders. The parceling of neuropil by astrocytes may have many interesting implications for our understanding of the functional interactions taking place between astrocytes and between astrocytes and neurons.

The infiltration of large regions of neuropil by individual astrocytes is a consequence of the unique morphology of protoplasmic astrocytes. The complex composition of the spongiform material of protoplasmic astrocytes has been examined in detail. Kosaka and Hama (1986) used high-voltage electron microscopic examination to demonstrate clearly that protoplasmic astrocytes can occupy extended spaces with a multitude of lamellar processes. More recently, Grosche et al. (1999) reported that, by using serial thin-section reconstructions through the spongiform processes of Bergmann glia (a specialized astrocyte of the cerebellum), it was possible to discern discreet "microdomains." These microdomains are very small in volume but appear to be capable of limiting the spread of glial calcium elevations in response to underlying neuronal activity in acute slices. It is possible that the fine processes of protoplasmic astrocytes of CA1 

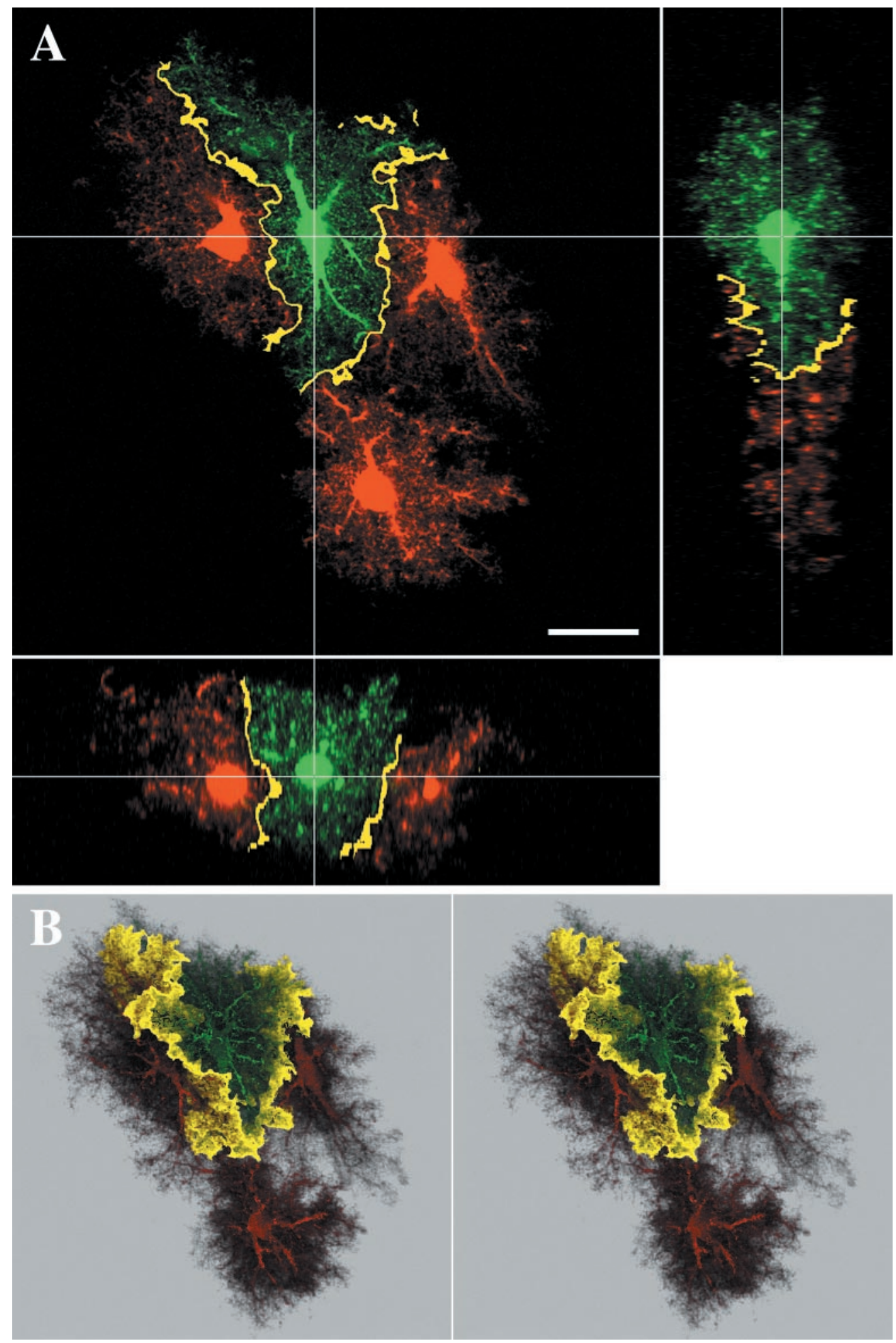

Figure 7. Another example of colocalization between adjacent astrocytes. $A$, Slice through adjacent astrocytes, as seen in Figure 6. B, Stereo pair of the same group of astrocytes. $3 \mathrm{D}$ views of colocalization reveal sheets in which neighboring astrocytes interact with each other. Scale bar, $20 \mu \mathrm{m}$. are similarly organized and that these astrocytes divide the neuropil on multiple levels.

Because of the inability to discriminate between the processes of neighboring astrocytes when these cells are metal impregnated or HRP labeled, previous work examining interastrocytic relationships relied on the assumption that the average astrocyte occupied a spherical region of neuropil (Rohlmann and Wolff, 1996). These assumptions led to the conclusion that astrocytes must interdigitate extensively and possess a very limited autocon- trol space. This was based on the examination of cortical astrocytes, and regional variations in astrocyte morphology are well known. We show that, in CA1, such assumptions would undoubtedly lead to an underestimation of the degree to which astrocytes independently invest neuropil. This is attributable to the large variation in morphology seen throughout the population of protoplasmic astrocytes within this region. Although the typical astrocyte in CA1 stratum radiatum is elongated parallel to the apical dendrites of CA1 pyramidal neurons, astrocytes were ob- 

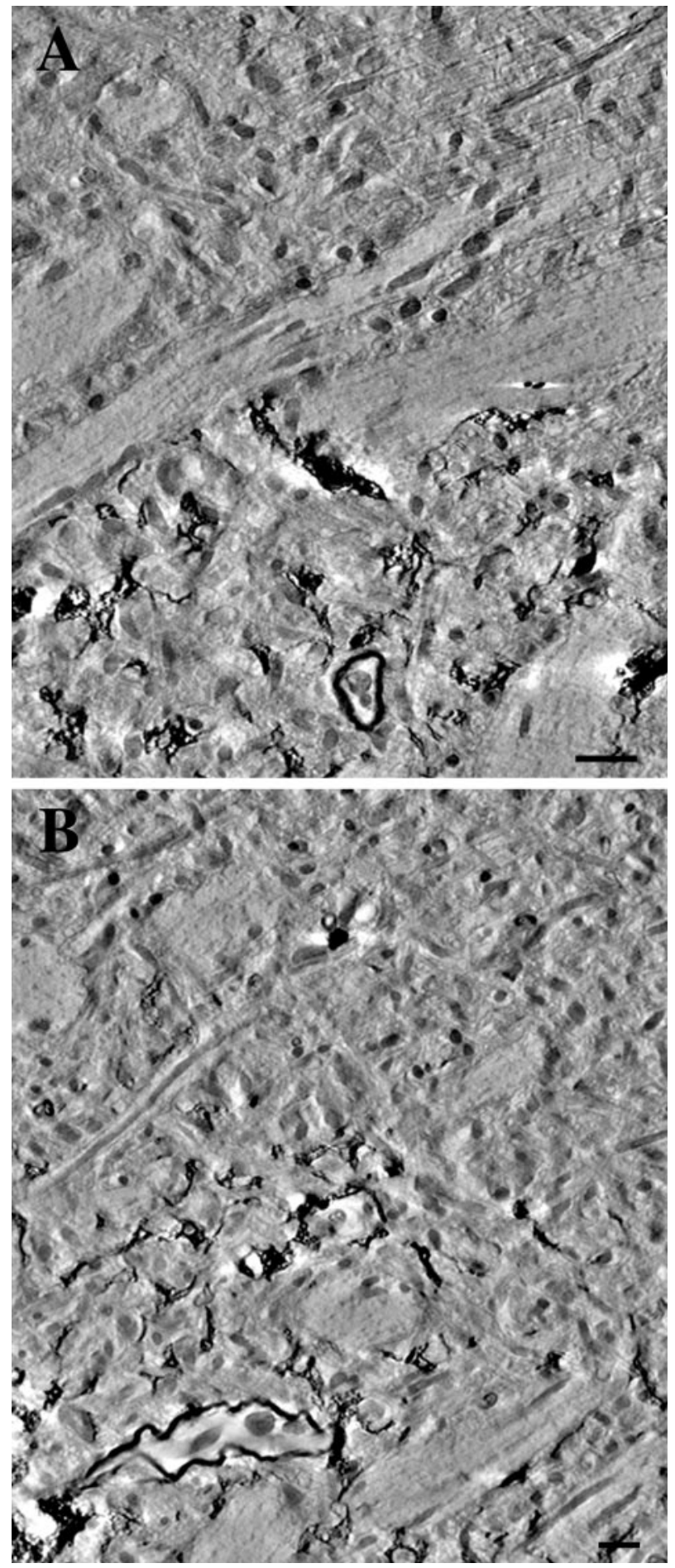

Figure 8. Electron microscopic examination of the boundary regions of a photooxidized astrocyte. $A, B$, Tomographic reconstructions of astrocyte boundary regions were generated using a $0.5-\mu \mathrm{m}$-thick section through a photoconverted astrocyte. Computational slices through the resulting volumes demonstrate an abrupt decrease in the density of fine astrocytic processes at the boundary to extent of the astrocyte. Scale bars, $1 \mu \mathrm{m}$.

served to range in size and overall shape, from approximately spherical to greatly elongated. This large variation in morphology allows astrocytes to fill all of the neuropil, without resorting to extensive overlap of their processes. Furthermore, although interdigitation does occur between the major processes of proto- plasmic astrocytes because of the highly ramified nature of the fine processes, astrocytes appear to minimize shared neuropilar volume. Indeed, overall, astrocyte processes appeared to avoid extensive interdigitation, resulting in the plethora of astrocyte morphologies observed. This phenomenon appears to result in the establishment of anatomical domains by astrocytes, in which the volume of solitary occupation by each astrocyte depends chiefly on the total neuropilar volume occupied by that astrocyte.

We have not yet determined whether the protoplasmic astrocytes of other regions parcel tissue as effectively as in CA1. Protoplasmic astrocytes vary in morphology between various gray matter regions. For example, in the molecular layer of dentate gyrus, we noticed that protoplasmic astrocytes are more likely than CA1 astrocytes to extend considerably longer, more divergent processes toward blood vessels. In the neostriatum, astrocytes tend to be more spherical in shape, as opposed to the generally prolate morphology seen in CA1 stratum radiatum (our unpublished observations). However, the "sponginess" of this cell type is conserved from region to region, and thus the parceling achieved in CA1 may well be maintained in other regions of the CNS.

\section{Contact spacing and the development of astrocyte arrays}

Our results are in large part consistent with the contact spacing model for the development of 3D astrocyte arrays in vivo (Distler et al., 1991; Tout et al., 1993). Astrocyte somata are usually well spaced from one another, and blood vessels appear to often have an influence on the organization of astrocytic processes and on overall astrocyte morphology (Chan-Ling and Stone, 1991). Although these results are consistent with the idea that astrocytes are evenly spaced throughout the neuropil, we have not found evidence of the extensive interdigitation or contact between large astrocyte processes described in previous reports. Stone and colleagues have suggested that contact spacing would allow astrocytes to create space between themselves and therefore obtain a homogeneous distribution of astrocytes throughout the tissue (Tout et al., 1993). Our results suggest that protoplasmic astrocytes do not simply create space between themselves but manage to establish exclusive territories for themselves.

Using GFAP immunolabeling, it has been demonstrated in developing stratum radiatum that GFAP-positive astrocytes initially show a dramatic increase in overall size until sometime after $24 \mathrm{~d}$ of age, when they appear to contract slightly to the size seen in adult rat (Nixdorf-Bergweiler et al., 1994). Furthermore, the degree of branching in GFAP-labeled processes and overlap between branches both decrease during this same interval. It is possible that these changes in GFAP distribution reflect the contribution of astrocytic dendrites to the process of establishing the $3 \mathrm{D}$ array. As stressed above, astrocytic arborization may not be reflected well by GFAP labeling alone. Astrocytes may use a mechanism for establishing astrocyte distribution that is independent of process extension and development.

\section{Astrocyte heterogeneity and anatomical domains}

Recent investigations have reported evidence for a subpopulation of GFAP-negative astrocytes in adult CA1 molecular layer (up to 40\% GFAP-negative) (Walz and Lang, 1998; Walz and Wuttke, 1999). It has been proposed that this heterogeneity in GFAP expression may be correlated with two electrophysiologically distinct classes of astrocytes in adult CA1: "passive" astrocytes, 
which express GFAP and lack voltage-dependant membrane currents, and "complex" astrocytes, which lack GFAP and express a range of voltage-dependant currents (Walz, 2000). Because the tissue used in our study was perfusion fixed, the in vivo state of GFAP distribution and astrocyte morphology should have been well preserved. Reports of both passive and complex astrocytes injected with LY depict cells with spongiform morphologies consistent with that of protoplasmic astrocytes (Jabs et al., 1997; Cotrina et al., 1998). This stated, we found no support for heterogeneity in GFAP expression among protoplasmic astrocytes in CA1. First, LY-filled, GFAP-stained protoplasmic astrocytes consistently displayed a GFAP cytoskeleton. There was little variation in the intensity and extent of GFAP labeling among the filled astrocytes. Unlike previous studies of GFAP distribution across astrocyte populations (Walz and Lang, 1998), our analysis did not include cells incompletely filled via dye transfer (astrocytes are known to form gap junctions with other cell types), but rather each cell was individually filled and clearly identified as a protoplasmic astrocyte. Second, having shown that astrocytes do not overlap extensively and that each immunolabeled GFAP cytoskeleton only represents $\sim 15 \%$ of the total astrocytic volume (using our protocol), it is difficult to see where additional GFAP-negative protoplasmic astrocytes could reside in typical GFAP-labeled CA1 (Fig. $2 C$ ). There appears to be no space remaining for GFAP-negative protoplasmic astrocytes. Although we conclude that it is unlikely that GFAP expression varies significantly between CA1 astrocytes, disparities in GFAP immunoreactivity are known to exist between astrocytes within certain regions of the CNS, such as the cortex (Stichel et al., 1991). These differences may very well reflect populations of astrocytes with distinct functional demands (Holthoff and Witte, 2000) or physiological states (Kafitz et al., 1999).

If indeed there are diverse types of protoplasmic astrocytes in adult CA1, the combination of functional heterogeneity and large volumes of solitary investment could have significant physiological implications. It would suggest that astrocytes subtend large groups of synapses and neuronal processes and that these clusters of neuronal elements are differentially influenced by the glial processes in which they are enmeshed, depending on which type of astrocyte is enveloping these structures. For example, it has been estimated that, in adult rat CA1, there are $\sim 213$ synapses/ $100 \mu \mathrm{m}^{3}$ (Kirov et al., 1999). Therefore, an astrocyte occupying $66,000 \mu \mathrm{m}^{3}$ of neuropil would oversee $\sim 140,000$ synapses. The vast majority of these synapses would be under the influence of this single astrocyte. Whether astrocytes compartmentalize dendrites and axons is not clear, but the longitudinal arrangement of many astrocytes with respect to apical CA1 dendrites suggest that this may also occur to a lesser extent. One wonders why such a structural arrangement exists if there is a division of labor between diverse forms of astrocytes. Alternatively, the physiological properties of astrocytes may be dynamic, in which the state of an individual astrocyte correlates to conditions in the local environment (Walz, 2000). Of course, the heterogeneity seen among astrocytes in CA1 using patch-clamp investigations in acute slices may simply be artifactual (Bordey and Sontheimer, 1998) or only present during development. Regardless, determination of the conditions under which long-distance versus short-distance signaling events occur within and between astrocytes will be necessary for attaining a full understanding of the functional implications of astrocyte domains.

\section{REFERENCES}

Araque A, Parpura V, Sanzgiri RP, Haydon PG (1999) Tripartite synapses: glia, the unacknowledged partner. Trends Neurosci 22:208-215.

Belichenko PV, Dahlström A (1995) Studies on the 3-dimensional architecture of dendritic spines and varicosities in human cortex by confocal laser scanning microscopy and Lucifer yellow microinjections. J Neurosci Methods 57:55-61.

Bignami A, Eng LF, Dahl D, Uyeda CT (1972) Localization of the glial fibrillary acidic protein in astrocytes by immunofluorescence. Brain Res 43:429-435.

Bordey A, Sontheimer H (1998) Passive glial cells, fact or artifact? J Membr Biol 166:213-222.

Buhl EH (1993) Intracellular injection in fixed slices in combination with neuroanatomical tracing techniques and electron microscopy to determine multisynaptic pathways in the brain. Microsc Res Tech 24:15-30.

Bushong E, Martone ME, Foster C, Ellisman MH (1999) Effectiveness of GFAP immunolabeling for estimating astrocytic volume. Microsc Microanal 5:1340-1341.

Chan-Ling T, Stone J (1991) Factors determining the migration of astrocytes into the developing retina: migration does not depend on intact axons or patent vessels. J Comp Neurol 303:375-386.

Connor JR, Berkowitz EM (1985) A demonstration of glial filament distribution in astrocytes isolated from rat cerebral cortex. Neuroscience 16:33-44.

Cotrina ML, Kang J, Lin JH, Bueno E, Hansen TW, He L, Liu Y, Nedergaard M (1998) Astrocytic gap junctions remain open during ischemic conditions. J Neurosci 18:2520-2537.

D'Ambrosio R, Wenzel J, Schwartzkroin PA, McKhann II GM, Janigro D (1998) Functional specialization and topographic segregation of hippocampal astrocytes. J Neurosci 18:4425-4438.

Deerinck TJ, Martone ME, Lev-Ram V, Green DP, Tsien RY, Spector DL, Huang S, Ellisman MH (1994) Fluorescence photooxidation with eosin: a method for high resolution immunolocalization and in situ hybridization detection for light and electron microscopy. J Cell Biol 126:901-910.

Distler C, Dreher Z, Stone J (1991) Contact spacing among astrocytes in the central nervous system: an hypothesis of their structural role. Glia 4:484-494.

Dreher Z, Tout S, Stone J (1994) Interactions of living astrocytes in vitro: evidence of the development of contact spacing. Glia 11:57-63.

Eng LF, Vanderhaeghen JJ, Bignami A, Gerstl B (1971) An acidic protein isolated from fibrous astrocytes. Brain Res 28:351-354.

Giberson RT, Demaree Jr RS, Nordhausen RW (1997) Four-hour processing of clinical/diagnostic specimens for electron microscopy using microwave technique. J Vet Diagn Invest 9:61-67.

Grosche J, Matyash V, Möller T, Verkhratsky A, Reichenbach A, Kettenmann H (1999) Microdomains for neuron-glia interaction: parallel fiber signaling to Bergmann glial cells. Nat Neurosci 2:139-143.

Harlow E, Lane D (1988) Antibodies: a laboratory manual, p 418. Cold Spring Harbor, NY: Cold Spring Harbor Laboratory.

Hatten ME, Mason CA (1990) Mechanisms of glial-guided neuronal migration in vitro and in vivo. Experientia 46:907-916.

Holthoff K, Witte OW (2000) Directed spatial potassium redistribution in rat neocortex. Glia 29:288-292.

Jabs R, Paterson IA, Walz W (1997) Qualitative analysis of membrane currents in glial cells from normal and gliotic tissue in situ: downregulation of $\mathrm{Na}^{+}$current and lack of $\mathrm{P} 2$ purinergic responses. Neuroscience 81:847-860.

Kafitz KW, Güttinger HR, Müller CM (1999) Seasonal changes in astrocytes parallel neuronal plasticity in the song control area $\mathrm{HVc}$ of the canary. Glia 27:88-100.

Keyser DO, Pellmar TC (1994) Synaptic transmission in the hippocampus: critical role for glial cells. Glia 10:237-243.

Kirov SA, Sorra KE, Harris KM (1999) Slices have more synapses than perfusion-fixed hippocampus from both young and mature rats. J Neurosci 19:2876-2886.

Kosaka T, Hama K (1986) Three-dimensional structure of astrocytes in the rat dentate gyrus. J Comp Neurol 249:242-260.

Levine JM, Reynolds R, Fawcett JW (2001) The oligodendrocyte precursor cell in health and disease. Trends Neurosci 24:39-47.

Maxwell DS, Kruger L (1965) The fine structure of astrocytes in the cerebral cortex and their response to focal injury produced by heavy ionizing particles. J Cell Biol 25:141-157.

Nixdorf-Bergweiler BE, Albrecht D, Heinemann U (1994) Developmental changes in the number, size, and orientation of GFAP-positive cells in the CA1 region of rat hippocampus. Glia 12:180-195.

Perkins GA, Renken CW, Song JY, Frey TG, Young SJ, Lamont S, Martone ME, Lindsey S, Ellisman MH (1997) Electron tomography of large, multicomponent biological structures. J Struct Biol 120:219-227.

Peters A, Palay SL, Webster H deF (1991) The fine structure of the nervous system, Ed 3, p 295. New York: Oxford UP. 
Rohlmann A, Wolff JR (1996) Subcellular topography and plasticity of gap junction distribution on astrocytes. In: Gap junctions in the nervous system (Spray DC, Dermietzel R, eds), pp 175-192. Austin, TX: Landes.

Schmidt-Kastner R, Szymas J (1990) Immunohistochemistry of glial fibrillary acidic protein, vimentin and S-100 protein for study of astrocytes in hippocampus of rat. J Chem Neuroanat 3:179-192.

Stichel CC, Müller CM, Zilles K (1991) Distribution of glial fibrillary acidic protein and vimentin immunoreactivity during rat visual cortex development. J Neurocytol 20:97-108.

Tout S, Dreher Z, Chan-Ling T, Stone J (1993) Contact-spacing among astrocytes is independent of neighbouring structures: in vivo and in vitro evidence. J Comp Neurol 332:433-443.

Ullian EM, Sapperstein SK, Christopherson KS, Barres BA (2001) Control of synapse number by glia. Science 291:657-661.
Vernadakis A (1996) Glia-neuron intercommunications and synaptic plasticity. Prog Neurobiol 49:185-214.

Walz W (1989) Role of glial cells in the regulation of the brain ion microenvironment. Prog Neurobiol 33:309-333.

Walz W (2000) Controversy surrounding the existence of discrete functional classes of astrocytes in adult gray matter. Glia 31:95-103.

Walz W, Lang MK (1998) Immunocytochemical evidence for a distinct GFAP-negative subpopulation of astrocytes in the adult rat hippocampus. Neurosci Lett 257:127-130.

Walz W, Wuttke WA (1999) Independent mechanisms of potassium clearance by astrocytes in gliotic tissue. J Neurosci Res 56:595-603.

Wolff JR (1976) The morphological organization of cortical neuroglia. In: Handbook of electroencephalography and clinical neurophysiology, Vol 2A (Remond A, Creutzfeldt O, eds), pp 26-43. Amsterdam: Elsevier. 